\title{
UNIQUENESS OF MEROMORPHIC FUNCTIONS WITH WEAKLY WEIGHTED SHARING
}

\author{
THAMIR ALZAHARY
}

(Received 21 May 2012; accepted 22 February 2013; first published online 28 June 2013)

\begin{abstract}
In this paper, we introduce the notion of weakly weighted sharing of zeros of meromorphic functions ignoring multiplicities, which extends the notion of weakly weighted sharing counting multiplicities, and we also introduce the notion of multiplicity. By using these notions, we prove some results on the uniqueness of meromorphic functions concerning differential polynomials sharing nonzero finite values. The results in this paper extend the results of Yang and Hua, Fang, and Dyavanal. In this paper, we correct defective points in the paper of $\mathrm{Wu}$ et al. ['Uniqueness of meromorphic functions sharing one value', Bull. Aust. Math. Soc. 85(2012), 280-294].
\end{abstract}

2010 Mathematics subject classification: primary 30D35.

Keywords and phrases: meromorphic function, differential polynomial, weakly weighted sharing.

\section{Introduction and main results}

Let $f$ and $g$ be nonconstant meromorphic functions in the complex plane $\mathbb{C}$. In this paper we will use the standard notation of value distribution theory of meromorphic functions, such as $T(r, f), N(r, f), m(r, f)$ and $\bar{N}(r, f)$ (see [3, 8]). The notation $S(r, f)$ is defined to be any quantity satisfying $S(r, f)=o(1)(T(r, f))$, possibly outside a set $E$ of finite Lebesgue measure. We let $S(r)$ denote any term which is both $S(r, f)$ and $S(r, g)$ simultaneously. A meromorphic function $a$ is said to be a small function of $f$ if $T(r, a)=S(r, f)$.

Let $k$ be a positive integer, and let $a$ be a small function of $f$. We denote by $N_{k}(r, f)$ the counting function of the poles of $f$ whose multiplicities are less than or equal to $k$, by $N_{(k}(r, f)$ the counting function of the poles of $f$ whose multiplicities are greater than or equal to $k$, and by $\bar{N}_{k)}(r, f)$ and $\bar{N}_{(k}(r, f)$ the reduced functions of $N_{k)}(r, f)$ and $N_{(k}(r, f)$, respectively. We let

$$
N_{k}(r, f)=\bar{N}(r, f)+\bar{N}_{(2}(r, f)+\cdots+\bar{N}_{(k}(r, f)
$$

(C) 2013 Australian Mathematical Publishing Association Inc. 0004-9727/2013 \$16.00 
and

$$
\Theta(a, f)=1-\limsup _{r \rightarrow \infty} \frac{\bar{N}\left(r, \frac{1}{f-a}\right)}{T(r, f)} .
$$

For $a \in \mathbb{C}$, we say that $f$ and $g$ share $a \mathrm{CM}$ (counting multiplicities) provided that $f-a$ and $g-a$ have the same zeros with the same multiplicities; if we do not consider multiplicities, then $f$ and $g$ are said to share $a$ IM (ignoring multiplicities). We say that $f$ and $g$ share $\infty \mathrm{CM}$ (respectively, IM) if $1 / f, 1 / g$ share $0 \mathrm{CM}$ (respectively, IM).

Lin and Lin [4] introduced the notion of weakly weighted sharing for $C M$, as given in the following definition.

Definition 1.1 [4]. Let $k$ be a positive integer or infinity, and let $a$ be a small function of nonconstant meromorphic functions $f$ and $g$. We denote by $\bar{N}_{k)}(r, a, f, g)$ the reduced counting function of those $a$-points of $f$, whose multiplicities are equal to the corresponding $a$-points of $g$, and both of their multiplicities are less than or equal to $k$. We say that $f$ and $g$ share $(a, k)^{*} \mathrm{CM}$, if

$$
\bar{N}_{k)}\left(r, \frac{1}{f-a}\right)+\bar{N}_{k)}\left(r, \frac{1}{g-a}\right)-2 \bar{N}_{k)}(r, a, f, g)=S(r) .
$$

We generalise the definitions of CM, IM and the weakly weighted sharing for CM to the weakly weighted sharing for IM as given by the following definition.

Definition 1.2. Let $k$ be a positive integer or infinity, and let $a$ be a small function of nonconstant meromorphic functions $f$ and $g$. We denote by $\bar{N}_{k)}(r, 1 /(f-a), g \neq a)$ the reduced counting function of those $a$-points of $f$ whose multiplicities are less than or equal to $k$, that are not the $a$-points of $g$. If

$$
\bar{N}_{k)}\left(r, \frac{1}{f-a}, g \neq a\right) \text { and } \quad \bar{N}_{k)}\left(r, \frac{1}{g-a}, f \neq a\right)=S(r),
$$

we say that $f$ and $g$ share $(a, k)^{*}$ IM.

We note that, if $f$ and $g$ share $(a, k)^{*} \mathrm{IM}$, then $f$ and $g$ share $(a, p)^{*} \mathrm{IM}$, for all integers $1 \leq p \leq k$. Also, we note that if $f$ and $g$ share $(a, k)^{*} \mathrm{CM}$, then $f$ and $g$ share $(a, k)^{*} \mathrm{IM}$.

In 1997, Yang and Hua [7] proved the following theorem.

THEOREM A. Let $f$ and $g$ be two nonconstant meromorphic functions, and $n \geq 11$ be a positive integer. If $f^{n} f^{\prime}$ and $g^{n} g^{\prime}$ share a $C M$, where $a \neq 0, \infty$ is a complex number, then either $f \equiv d g$, for some $(n+1)$ th root of unity $d$, or $f(z) \equiv c_{1} e^{c z}$ and $g(z) \equiv c_{2} e^{-c z}$, where $c_{1}, c_{2}$ and c are three constants satisfying $\left(c_{1} c_{2}\right)^{n} c^{2}=-a$.

Note that $f^{n} f^{\prime}=(1 /(n+1))\left(f^{n+1}\right)^{\prime}$. 
In 2002, Fang [2] proved the following results.

TheOREM B. Let $f$ and $g$ be two nonconstant entire functions, and let $n$ and $k$ be two positive integers satisfying $n \geq 2 k+8$. If $\left(f^{n}(f-1)\right)^{(k)}$ and $\left(g^{n}(g-1)\right)^{(k)}$ share $1 C M$, then $f \equiv g$.

Theorem C. Let $f$ and $g$ be two nonconstant entire functions, and let $n$ and $k$ be two positive integers satisfying $n \geq 2 k+4$. If $\left(f^{n}\right)^{(k)}$ and $\left(g^{n}\right)^{(k)}$ share $1 \mathrm{CM}$, then either $f(z) \equiv c_{1} e^{c z}$ and $g(z) \equiv c_{2} e^{-c z}$, where $c_{1}, c_{2}$ and $c$ are three constants satisfying $(-1)^{k}\left(c_{1} c_{2}\right)^{n}(n c)^{2 k}=1$, or $f(z) \equiv \operatorname{tg}(z)$, for a constant $t$ such that $t^{n}=1$.

In 2011, Dyavanal [1] proved the following two theorems, by using the notion of multiplicity.

THEOREM D. Let $f$ and $g$ be two nonconstant meromorphic functions, whose zeros and poles are of multiplicities at least $s$, where $s$ is a positive integer. Let $n \geq 2$ be an integer satisfying $(n+1) s \geq 12$. If $f^{n} f^{\prime}$ and $g^{n} g^{\prime}$ share $1 C M$, then either $f(z) \equiv d g(z)$, for some $(n+1)$ th root of unity $d$, or $f(z) \equiv c_{1} e^{c z}$ and $g(z) \equiv c_{2} e^{-c z}$, where $c_{1}, c_{2}$ and $c$ are three constants satisfying $\left(c_{1} c_{2}\right)^{n+1} c^{2}=-1$.

THEOREM E. Let $f$ and $g$ be two nonconstant distinct meromorphic functions, whose zeros and poles are of multiplicities at least $s$, where $s$ is a positive integer. Let $n$ be an integer satisfying $(n-1) s \geq 5$. If $f^{n}(f-1) f^{\prime}$ and $g^{n}(g-1) g^{\prime}$ share $1 C M$, then

$$
g(z)=\frac{(n+2)\left(1-h^{n+1}\right)}{(n+1)\left(1-h^{n+2}\right)}, \quad f(z)=h \frac{(n+2)\left(1-h^{n+1}\right)}{(n+1)\left(1-h^{n+2}\right)},
$$

where $h$ is a nonconstant meromorphic function.

Note that $f^{n}(f-1) f^{\prime}=\left(a f^{n+1}(f+b)\right)^{\prime}$, where $a=1 /(n+2)$ and $b=-(n+2) /(n+1)$.

Dyavanal raised the question in his paper whether the differential polynomials in Theorem $\mathrm{D}$ (respectively, Theorem $\mathrm{E}$ ) can be replaced by the form $\left(f^{n}\right)^{(k)}$ (respectively, $\left.\left(f^{n}(f-1)\right)^{(k)}\right)$, where $k \geq 1$ is an integer, and whether a CM shared valued can be replaced by an IM shared value in Theorems D and E.

In 2012, Wu et al. [6] claimed to prove the following two theorems to answer Dyavanal's question.

THEOREM F [6, Theorem 1.1]. Let $f$ and $g$ be two nonconstant meromorphic functions, whose zeros and poles are of multiplicities at least $\ell$, where $\ell$ is a positive integer. Let $n \geq 2 k+1$ be an integer satisfying $n \ell \geq 7 k+12$. If $\left(f^{n}\right)^{(k)}$ and $\left(g^{n}\right)^{(k)}$ share 1 IM, then either $f(z) \equiv \operatorname{tg}(z)$, for a constant $t$ such that $t^{n}=1$, or $f(z) \equiv c_{1} e^{c z}$ and $g(z) \equiv c_{2} e^{-c z}$, where $c_{1}, c_{2}$ and c are three constants satisfying $(-1)^{k}\left(c_{1} c_{2}\right)^{n}(n c)^{2 k}=1$.

Theorem G [6, Theorem 1.2]. Let $f$ and $g$ be two nonconstant meromorphic functions, whose zeros and poles are of multiplicities at least $l<(7 k / 2)+7$, where $l$ is a positive integer. Let $n$ be an integer satisfying $(n+1) l \geq 7 k+17$. If $\left(f^{n}(f-1)\right)^{(k)}$ and $\left(g^{n}(g-1)\right)^{(k)}$ share $1 I M$, and $\Theta(\infty, f)>2 / n$, then $f \equiv g$. 
REMARK 1.3. There are some gaps in Wu et al.'s proofs of Theorems F and G. The first mistake is in the proof of [6, Lemma 2.4]. Wu et al. claimed that ' $\Phi(z)$ has poles, all simple, only at zeros of $f^{(k+1)}$ and $g^{(k+1)}$ and poles of $f$ and $g$, and 1-points of $f$ whose multiplicities are not equal to the multiplicities of the corresponding 1-points of $g$ '.

Indeed, the function $\Phi(z)$ may also have other poles, for example, the zeros of $f^{(k+1)}$ which are the zeros of $f$ with multiplicities less than $k+1$, and hence these zeros are not counted in $\bar{N}_{(k+2}(r, 1 / f)$. In other words, the terms $\bar{N}_{(k+2}(r, 1 / f)$ and $\bar{N}_{(k+2}(r, 1 / g)$ in the inequality for $N(r, \Phi)$ should be $\bar{N}(r, 1 / f)$ and $\bar{N}(r, 1 / g)$, respectively.

The second mistake is in the proof of Theorem F. It is the claim, without proof, that if $f$ and $g$ satisfy (3.1), then $f$ and $g$ have no poles. In fact, probably $f$ or $g$ has poles.

The third mistake is in the proof of Theorem $\mathrm{G}$ and is similar to that in the proof of Theorem F. Thus Theorems F and G have defects in their proofs.

Question 1.4. Let $f$ and $g$ be two transcendental meromorphic functions, and let $\alpha_{1}$ and $\alpha_{2}$ be nonzero small functions of $f$ and $g$. What happens if $\left(\alpha_{2} f^{n}\left(f^{m}+\alpha_{1}\right)\right)^{(k)}$ and $\left(\alpha_{2} g^{n}\left(g^{m}+\alpha_{1}\right)\right)^{(k)}$ share $(1,1)^{*}$ IM or CM? What happens if $\left(\alpha_{1} f^{n}\right)^{(k)}$ and $\left(\alpha_{2} g^{n}\right)^{(k)}$ share $(1,1)^{*}$ IM or CM?

We first introduce the new notion of multiplicity, given by the following definition.

Definition 1.5. Let $f$ and $g$ be two meromorphic functions, and let $\alpha$ be a finite set of small functions of $f$ and $g$. Let $S$ be a set of the poles of $f$ which are not the poles or zeros of any element in $\alpha$. If $S$ is nonempty, we denote by $n_{\alpha}(\infty, f)$ the minimal multiplicity of those poles of $f$ that belong to $S$, and by $n_{\alpha}(\infty, f)=0$ if $S$ is empty. Define $n_{\alpha}(\infty, f, g)=\min \left\{n_{\alpha}(\infty, f), n_{\alpha}(\infty, g)\right\}$. We denote the symbol $n_{\alpha}(\infty, 1 / f, 1 / g)$ by $n_{\alpha}(0, f, g)$. In particular, if $\alpha$ consists of only complex numbers, then $n_{\alpha}(\infty, f, g)$ is the minimal multiplicity of those poles of $f$ and $g$.

In this paper, we use the notion of weakly weighted sharing and the notion of multiplicity to answer Question 1.4 and correct Theorems F and G.

THeOREM 1.6. Let $\alpha$ and $\phi$ be two nonempty finite sets of small functions of two transcendental meromorphic functions $f$ and $g$, and let $n, m$ and $k$ be three positive integers with $n+m>2 k$ and $n \geq k+1$. Let $\alpha_{1}$ and $\alpha_{2}$ be two nonzero elements in $\alpha \bigcap \phi$. Suppose that $\left(\alpha_{2} f^{n}\left(f^{m}+\alpha_{1}\right)\right)^{(k)}$ and $\left(\alpha_{2} g^{n}\left(g^{m}+\alpha_{1}\right)\right)^{(k)}$ share $(1,1)^{*}$ IM. Assume also that the following three conditions hold.

(A1) $(n-4 m) t_{1} t_{2}>(5 k+7) t_{1}+(4 k+7) t_{2}$, where $t_{1}=\max \left\{n_{\alpha}(\infty, f, g), 1\right\}$ and $t_{2}=$ $\max \left\{n_{\phi}(0, f, g), 1\right\}$.

(A2) If $\left(\alpha_{2} f^{n}\left(f^{m}+\alpha_{1}\right)\right)^{(k)}\left(\alpha_{2} g^{n}\left(g^{m}+\alpha_{1}\right)\right)^{(k)} \equiv 1$, then

$$
\frac{m}{n+m-2 k}<\limsup _{r \rightarrow \infty} \frac{s_{g} \bar{N}(r, g)+s_{f} \bar{N}(r, f)}{\left(1+s_{g}\right) T(r, f)+\left(1+s_{f}\right) T(r, g)},
$$

where $s_{g}$ (respectively, $s_{f}$ ) is the minimal multiplicity of those poles of $g$ (respectively, $f$ ) which are not the zeros of $f$ (respectively, $g$ ). 
(A3) Either $m t_{1} t_{2} \geq 2$, or else if $m t_{1} t_{2}=1$ then

$$
\frac{2}{n}<\max \{\Theta(\infty, g)+\Theta(0, g), \Theta(\infty, f)+\Theta(0, f)\} .
$$

Then $f \equiv c g$, where $c$ is a nonzero constant satisfying $c^{q}=1$; here, $q=\operatorname{gcd}(n, m)$.

From Theorem 1.6, we correct Theorem G by the following corollary.

Corollary 1.7. Let $f$ and $g$ be two transcendental meromorphic functions, whose zeros and poles are of multiplicities at least $t$, where $t$ is a positive integer. Let $n$ and $k$ be two positive integers satisfying $n \geq 2 k$ and $(n-4) t>9 k+14$. Assume that the following two conditions hold.

(i) If $\left(f^{n}(f-1)\right)^{(k)}\left(g^{n}(g-1)\right)^{(k)} \equiv 1$, then

$$
\frac{1}{n-2 k+1}<\limsup _{r \rightarrow \infty} \frac{s_{g} \bar{N}(r, g)+s_{f} \bar{N}(r, f)}{\left(1+s_{g}\right) T(r, f)+\left(1+s_{f}\right) T(r, g)},
$$

where $s_{g}$ (respectively, $s_{f}$ ) is the minimal multiplicity of those poles of $g$ (respectively, $f$ ) which are not the zeros of $f$ (respectively, $g$ ).

(ii) Either $t \geq 2$, or else if $t=1$ then

$$
\frac{2}{n}<\max \{\Theta(\infty, g)+\Theta(0, g), \Theta(\infty, f)+\Theta(0, f)\} .
$$

If $\left(f^{n}(f-1)\right)^{(k)}$ and $\left(g^{n}(g-1)\right)^{(k)}$ share 1 IM, then $f \equiv g$.

THeorem 1.8. Let $\alpha$ and $\phi$ be two nonempty finite sets of small functions of two transcendental meromorphic functions $f$ and $g$, and let $n, m$ and $k$ be three positive integers with $n+m>2 k$ and $n \geq k+1$. Let $\alpha_{1}$ and $\alpha_{2}$ be two nonzero elements in $\alpha \bigcap \phi$. Suppose that $\left(\alpha_{2} f^{n}\left(f^{m}+\alpha_{1}\right)\right)^{(k)}$ and $\left(\alpha_{2} g^{n}\left(g^{m}+\alpha_{1}\right)\right)^{(k)}$ share $\left(1, \ell_{1}\right)^{*} C M$ and $\left(1, \ell_{2}\right)^{*} I M$, where $\ell_{1}$ and $\ell_{2}$ are positive integers with $\ell_{2} \geq \ell_{1}$. Assume that the conditions (A2) and (A3) in Theorem 1.8 hold. Then the conclusion of Theorem 1.6 remains valid if at least one of the following conditions holds.

(B1) $(n-m) t_{1} t_{2}>(k+4) t_{2}+2(k+2) t_{1}+\ell\left((k+1)\left(t_{1}+t_{2}\right)+m t_{1} t_{2}\right)$.

(B2) $(n-m) t_{1} t_{2}>(k+4) t_{2}+2(k+2) t_{1}$ with $\ell_{1} \geq 2, \ell_{2} \geq 3$.

Here, $t_{1}$ and $t_{2}$ are as defined in Theorem 1.6 and

$$
\ell=\left\{\begin{array}{cl}
2 & \text { if } \ell_{1}=\ell_{2}=1 \\
\frac{1}{2}+\frac{1}{\ell_{2}} & \text { if } \ell_{1}=1, \ell_{2} \geq 2 \\
\frac{2}{\ell_{2}} & \text { if } \ell_{1} \geq 2 .
\end{array}\right.
$$

THEOREM 1.9. Let $\alpha$ and $\phi$ be two nonempty finite sets of small functions of two transcendental meromorphic functions $f$ and $g$, and let $n$ and $k$ be two positive integers with $n \geq 2 k+1$. Let $\alpha_{1}$ and $\alpha_{2}$ be two nonzero elements in $\alpha \bigcap \phi$ such that $\alpha_{1} f^{n} \not \equiv \alpha_{2} g^{n}$. Suppose that $\left(\alpha_{1} f^{n}\right)^{(k)}$ and $\left(\alpha_{2} g^{n}\right)^{(k)}$ share $(1,1)^{*}$ IM and the following condition holds. 
(C1) $n t_{1} t_{2}>(5 k+7) t_{1}+(4 k+7) t_{2}$, where $t_{1}$ and $t_{2}$ are as defined in Theorem 1.6.

Then $\left(\alpha_{1} f^{n}\right)^{(k)}\left(\alpha_{2} g^{n}\right)^{(k)} \equiv 1, f^{n} \equiv a g^{-n}$ and $\bar{N}(r, f)+\bar{N}(r, 1 / f)=S(r)$, where a is a small function of $f$ and $g$. If, in addition, $\alpha$ and $\phi$ consist of only complex constants then $f$ and $g$ have no zeros.

From Theorem 1.9, we correct Theorem F by the following corollary.

COROLlary 1.10. Let $f$ and $g$ be two transcendental meromorphic functions, whose zeros and poles are of multiplicities at least $t$, where $t$ is a positive integer. Let $n$ and $k$ be two positive integers satisfying $n \geq 2 k+1$ and $n t>9 k+14$. If $\left(f^{n}\right)^{(k)}$ and $\left(g^{n}\right)^{(k)}$ share 1 IM, then either: (i) $f(z) \equiv d g(z)$, for a constant $d$ such that $d^{n}=1$; or else (ii) $f$ and $g$ have no zeros, and $\left(f^{n}\right)^{(k)}\left(g^{n}\right)^{(k)} \equiv 1, f^{n} \equiv a g^{-n}$ and $\bar{N}(r, f)=S(r)$, where a is a small function of $f$ and $g$.

THeOREM 1.11. Let $\alpha$ and $\phi$ be two nonempty finite sets of small functions of two transcendental meromorphic functions $f$ and $g$, and let $n$ and $k$ be two positive integers with $n \geq 2 k+1$. Let $\alpha_{1}$ and $\alpha_{2}$ be two nonzero elements in $\alpha \bigcap \phi$ such that $\alpha_{1} f^{n} \not \equiv \alpha_{2} g^{n}$. Suppose that $\left(\alpha_{1} f^{n}\right)^{(k)}$ and $\left(\alpha_{2} g^{n}\right)^{(k)}$ share $\left(1, \ell_{1}\right)^{*} C M$ and $\left(1, \ell_{2}\right)^{*} I M$, where $\ell_{1}$ and $\ell_{2}$ are positive integers with $\ell_{2} \geq \ell_{1}$. Then the conclusions of Theorem 1.9 remain valid if at least one of the following conditions holds.

(D1) $n t_{1} t_{2}>(k+4) t_{2}+2(k+2) t_{1}+\ell(k+1)\left(t_{1}+t_{2}\right)$.

(D2) $n t_{1} t_{2}>(k+4) t_{2}+2(k+2) t_{1}$ with $\ell_{1} \geq 2, \ell_{2} \geq 3$.

Here, $t_{1}$ and $t_{2}$ are as defined in Theorem 1.6 and $\ell$ is as defined in Theorem 1.8.

REMARK 1.12. The integer $\ell_{1}$ or $\ell_{2}$ in Theorems 1.8 and 1.11 can be replaced by $\infty$, in which case if $\ell_{2} \rightarrow \infty$, then $\ell$ becomes $\ell=0$ if $\ell_{1} \geq 2$, and $\ell=1 / 2$ if $\ell_{1}=1$.

\section{Lemmas}

LeMma 2.1. Let $f$ and $g$ be two nonconstant meromorphic functions such that $f g \not \equiv 1$ and $f \not \equiv g$. If $f$ and $g$ share $(1,1)^{*} I M$, then

$$
\begin{aligned}
T(r, g) \leq N_{2}(r, f)+N_{2}\left(r, \frac{1}{f}\right)+N_{2}(r, g)+N_{2}\left(r, \frac{1}{g}\right) \\
+2 \bar{N}(r, g)+2 \bar{N}\left(r, \frac{1}{g}\right)+\bar{N}(r, f)+\bar{N}\left(r, \frac{1}{f}\right)+S(r) .
\end{aligned}
$$

The result holds true if $f$ and $g$ are interchanged.

Proof. Set $H=\left(f^{\prime \prime} / f^{\prime}-2 f^{\prime} /(f-1)\right)-\left(g^{\prime \prime} / g^{\prime}-2 g^{\prime} /(g-1)\right)$. Suppose that $H \not \equiv 0$. If $z_{0}$ is a common simple zero of $f-1$ and $g-1$, then $z_{0}$ must be a zero point of $H$. Therefore, if we denote by $\bar{N}_{E}^{1)}(r, 1 /(f-1))$ the counting function of the common simple 1-points of $f$ and $g$, then, by the first fundamental theorem, we get $\bar{N}_{E}^{1)}(r, 1 /(f-1)) \leq N(r, H)+S(r)$. 
We can easily verify that possible poles of $H$ occur at: (i) multiples poles of $f$ and $g$; (ii) multiples zeros of $f$ and $g$; (iii) zeros of $f-1$ which are not the zeros of $g-1$; (iv) zeros of $g-1$ which are not the zeros of $f-1$; (v) common zeros of $f-1$ and $g-1$ such that the multiplicity of the 1-point of $f$ is not equal to the multiplicity of the corresponding 1-point of $g$; (vi) zeros of $f^{\prime}$ which are not the zeros of $f(f-1)$; (vii) zeros of $g^{\prime}$ which are not the zeros of $g(g-1)$.

We denote by $\bar{N}(r, 1 /(f-1), g \neq 1), \quad \bar{N}(r, 1 /(g-1), f \neq 1), \quad \bar{N}_{*}(r, 1, f, g)$, $\bar{N}_{0}\left(r, 1 / f^{\prime}\right)$ and $\bar{N}_{0}\left(r, 1 / g^{\prime}\right)$ the reduced counting functions of those points which correspond to (iii)-(vii), respectively. Then from the preceding explanation, we deduce that

$$
\begin{aligned}
\bar{N}_{E}^{1)}(r, 1 /(f-1)) \leq & \bar{N}_{(2}(r, f)+\bar{N}_{(2}(r, g)+\bar{N}_{(2}\left(r, \frac{1}{f}\right) \\
& +\bar{N}_{(2}\left(r, \frac{1}{g}\right)+\bar{N}_{*}(r, 1, f, g)+\bar{N}_{0}\left(r, \frac{1}{f^{\prime}}\right) \\
& +\bar{N}_{0}\left(r, \frac{1}{g^{\prime}}\right)+\bar{N}\left(r, \frac{1}{f-1}, g \neq 1\right)+\bar{N}\left(r, \frac{1}{g-1}, f \neq 1\right)+S(r) .
\end{aligned}
$$

By the second fundamental theorem,

$$
\begin{array}{r}
T(r, f)+T(r, g) \leq \bar{N}(r, f)+\bar{N}(r, g)+\bar{N}\left(r, \frac{1}{f}\right)+\bar{N}\left(r, \frac{1}{g}\right)+\bar{N}\left(r, \frac{1}{f-1}\right) \\
+\bar{N}\left(r, \frac{1}{g-1}\right)-\bar{N}_{0}\left(r, \frac{1}{f^{\prime}}\right)-\bar{N}_{0}\left(r, \frac{1}{g^{\prime}}\right)+S(r, f) .
\end{array}
$$

If we denote by $\bar{N}_{L 1}(r, 1 /(g-1))$ the reduced counting function of the common points of the simple 1-points of $f$ and the multiple 1-points of $g$ then, from the hypotheses that $f$ and $g$ share $(1,1)^{*} \mathrm{IM}$ and the first fundamental theorem, it is not difficult to show that

$$
\begin{aligned}
\bar{N}\left(r, \frac{1}{g-1}\right)+\bar{N}\left(r, \frac{1}{f-1}\right) \leq & \bar{N}_{E}^{1)}\left(r, \frac{1}{f-1}\right)+N_{2}\left(r, \frac{1}{f-1}\right)+\bar{N}_{L 1}\left(r, \frac{1}{g-1}\right) \\
& +\bar{N}\left(r, \frac{1}{g-1}, f \neq 1\right)-\bar{N}\left(r, \frac{1}{f-1}, g \neq 1\right)+S(r) \\
\leq & \bar{N}_{E}^{1)}\left(r, \frac{1}{f-1}\right)+T(r, f)+\bar{N}_{L 1}\left(r, \frac{1}{g-1}\right)+N_{2}\left(r, \frac{1}{f-1}\right) \\
& +\bar{N}\left(r, \frac{1}{g-1}, f \neq 1\right)-\bar{N}\left(r, \frac{1}{f-1}, g \neq 1\right) \\
& -N\left(r, \frac{1}{f-1}\right)+S(r) .
\end{aligned}
$$

It follows, from this, (2.1) and (2.2) that

$$
T(r, g) \leq N_{2}(r, f)+N_{2}\left(r, \frac{1}{f}\right)+N_{2}(r, g)+N_{2}\left(r, \frac{1}{g}\right)+J_{g}(r),
$$


where

$$
\begin{aligned}
J_{g}(r)=\bar{N}_{*} & (r, 1, f, g)+\bar{N}_{L 1}\left(r, \frac{1}{g-1}\right)+2 \bar{N}\left(r, \frac{1}{g-1}, f \neq 1\right)-N\left(r, \frac{1}{f-1}\right) \\
& +N_{2}\left(r, \frac{1}{f-1}\right)+S(r) .
\end{aligned}
$$

Since $f$ and $g$ share $(1,1)^{*}$ IM, we see that

$$
\begin{aligned}
\bar{N}_{*}(r, 1, f, g)+\bar{N}\left(r, \frac{1}{g-1}, f \neq 1\right) & \leq \bar{N}\left(r, \frac{f^{\prime}}{f}\right)+\bar{N}\left(r, \frac{g^{\prime}}{g}\right)+S(r) \\
& =\bar{N}(r, g)+\bar{N}\left(r, \frac{1}{g}\right)+\bar{N}(r, f)+\bar{N}\left(r, \frac{1}{f}\right)+S(r) .
\end{aligned}
$$

In the same manner,

$$
\bar{N}_{L 1}\left(r, \frac{1}{g-1}\right)+\bar{N}\left(r, \frac{1}{g-1}, f \neq 1\right) \leq \bar{N}\left(r, g^{\prime} / g\right)+S(r)=\bar{N}(r, g)+\bar{N}\left(r, \frac{1}{g}\right)+S(r) .
$$

From this inequality and (2.3)-(2.5), we obtain the inequality stated in the lemma. In the same way, we can get an inequality similar to that stated by interchanging $f$ and $g$. This proves Lemma 2.1 when $H \not \equiv 0$. In the next lemma, we will prove that Lemma 2.1 is clear when $H \equiv 0$.

Lemma 2.2. Suppose, in addition to the assumptions of Lemma 2.1, that $f$ and $g$ share $\left(1, \ell_{1}\right)^{*} C M$ and $\left(1, \ell_{2}\right)^{*} I M$, where $\ell_{1}$ and $\ell_{2}$ are positive integers with $\ell_{2} \geq \ell_{1}$. Then

$$
\begin{gathered}
T(r, g) \leq N_{2}(r, f)+N_{2}\left(r, \frac{1}{f}\right)+N_{2}(r, g)+N_{2}\left(r, \frac{1}{g}\right) \\
+\ell\left(\bar{N}(r, g)+\bar{N}\left(r, \frac{1}{g}\right)\right)+S(r),
\end{gathered}
$$

where: (i) $\ell=2$, if $\ell_{1}=\ell_{2}=1$; (ii) $\ell=1 / 2+1 / \ell_{2}$, if $\ell_{1}=1, \ell_{2} \geq 2$; (iii) $\ell=2 / \ell_{2}$, if $\ell_{1} \geq 2$. Inequality (2.6) holds true, if $f$ and $g$ are interchanged. Moreover, if $\ell_{1} \geq 2$ and $\ell_{2} \geq 3$, then

$$
\frac{1}{2}(T(r, g)+T(r, f)) \leq N_{2}(r, f)+N_{2}\left(r, \frac{1}{f}\right)+N_{2}(r, g)+N_{2}\left(r, \frac{1}{g}\right)+S(r) .
$$

Proof. We repeat the same steps as in the proof of Lemma 2.1, and suppose that $H \not \equiv 0$. Since $f$ and $g$ share $(1,1)^{*}$ IM, the proof of Lemma 2.2 follows from (2.3) and (2.4). From the assumptions of Lemma 2.2, we deduce that $\bar{N}_{L 1}(r, 1 /(g-1))=S(r)$.

Since $\ell_{1} \geq 1$, we deduce from (2.4) that $J_{g}(r) \leq 2 N\left(r, g^{\prime} / g\right)+S(r)$; and if we suppose that $\ell_{2} \geq 2$ and $\ell_{1}=1$, then $\bar{N}(r, 1 /(g-1), f \neq 1) \leq\left(1 / \ell_{2}\right) N\left(r, g^{\prime} / g\right)+S(r)$ and

$$
J_{g}(r) \leq \bar{N}\left(r, \frac{1}{g-1}, f \neq 1\right)+\frac{1}{2} N\left(r, \frac{g^{\prime}}{g}\right)+S(r),
$$

which implies $J_{g}(r) \leq\left(1 / 2+1 / \ell_{2}\right) N\left(r, g^{\prime} / g\right)+S(r)$.

However, it is evident that $J_{g}(r) \leq\left(2 / \ell_{2}\right) N\left(r, g^{\prime} / g\right)+S(r)$, if $\ell_{1} \geq 2$ (in view of $\left.\ell_{2} \geq \ell_{1}\right)$. 
From the preceding illustration, (2.3) and (2.4), we get inequality (2.6). In same way, we can prove that inequality (2.6) is clear, by interchanging $f$ and $g$.

We now prove the last part of Lemma 2.2 when $\ell_{1} \geq 2$ and $\ell_{2} \geq 3$. From (2.4), $J_{g}(r)$ can be rewritten as

$$
J_{g}(r)=\bar{N}_{*}(r, 1, f, g)+2 \bar{N}\left(r, \frac{1}{g-1}, f \neq 1\right)-N\left(r, \frac{1}{f-1}\right)+N_{2}\left(r, \frac{1}{f-1}\right)+S(r) .
$$

Similarly,

$$
J_{f}(r)=\bar{N}_{*}(r, 1, f, g)+2 \bar{N}\left(r, \frac{1}{f-1}, g \neq 1\right)-N\left(r, \frac{1}{g-1}\right)+N_{2}\left(r, \frac{1}{g-1}\right)+S(r)
$$

We aim to prove the last part of Lemma 2.2; and that will follow if $J_{g}(r)+J_{f}(r) \leq$ $S(r)$. Since $f$ and $g$ share $\left(1, \ell_{1}\right)^{*} \mathrm{CM}$ and $\left(1, \ell_{2}\right)^{*} \mathrm{IM}$, we see that

$$
\begin{aligned}
& 2 \bar{N}_{*}(r, 1, f, g)+2 \bar{N}\left(r, \frac{1}{g-1}, f \neq 1\right)+2 \bar{N}\left(r, \frac{1}{f-1}, g \neq 1\right) \\
& \quad \leq N\left(r, \frac{1}{f-1}\right)-N_{2}\left(r, \frac{1}{f-1}\right)+N\left(r, \frac{1}{g-1}\right)-N_{2}\left(r, \frac{1}{g-1}\right),
\end{aligned}
$$

which implies $J_{g}(r)+J_{f}(r) \leq S(r)$. This proves Lemma 2.2 when $H \not \equiv 0$.

Suppose that $H=\left(f^{\prime \prime} / f^{\prime}-2 f^{\prime} /(f-1)\right)-\left(g^{\prime \prime} / g^{\prime}-2 g^{\prime} /(g-1)\right) \equiv 0$. Then

$$
f=\frac{(\alpha+1) g+\beta-\alpha-1}{\alpha g+\beta-\alpha} \quad \text { and } \quad g=\frac{(\alpha-\beta) f+\beta-\alpha-1}{\alpha f-(\alpha+1)},
$$

where $\alpha$ and $\beta$ are complex numbers. We check Lemmas 2.1 and 2.2 in the case $H \equiv 0$. To show that, it suffices to prove

$$
\max \{T(r, f), T(r, g)\} \leq N_{2}(r, f)+N_{2}\left(r, \frac{1}{f}\right)+N_{2}(r, g)+N_{2}\left(r, \frac{1}{g}\right)+S(r) .
$$

We now consider the following cases.

Case 1. Let $\alpha=0$. Since $f \not \equiv g, \beta \neq 1$. Consequently, from $(2.7), \bar{N}(r, 1 / f)=$ $\bar{N}(r, 1 /(g+\beta-1))+S(r)$, and $\bar{N}(r, 1 / g)=\bar{N}(r, 1 /(f-(\beta-1) / \beta))+S(r)$. It follows from this and the second fundamental theorem that

$$
\begin{aligned}
T(r, g) & \leq \bar{N}(r, g)+\bar{N}\left(r, \frac{1}{g}\right)+\bar{N}\left(r, \frac{1}{g+\beta-1}\right)+S(r) \\
& \leq \bar{N}(r, g)+\bar{N}\left(r, \frac{1}{g}\right)+\bar{N}\left(r, \frac{1}{f}\right)+S(r) .
\end{aligned}
$$


In the same way,

$$
T(r, f) \leq \bar{N}(r, f)+\bar{N}\left(r, \frac{1}{f}\right)+\bar{N}\left(r, \frac{1}{g}\right)+S(r) .
$$

From (2.9) and (2.10), we deduce (2.8).

Case 2. Let $\alpha \neq 0$. From (2.7),

$$
\bar{N}(r, f)=\bar{N}\left(r, \frac{1}{\alpha g+\beta-\alpha}\right)+S(r) \quad \text { and } \quad \bar{N}(r, g)=\bar{N}\left(r, \frac{1}{\alpha f-(\alpha+1)}\right)+S(r)
$$

If $\alpha \neq-1$ and $\alpha \neq \beta$ then, by proceeding in the same manner as in Case 1, we will arrive at (2.8).

Subcase 2.1. $\alpha=\beta$. Since $f g \not \equiv 1$, we deduce that $\alpha \neq-1$, and then from (2.7) we conclude that $\bar{N}(r, 1 / f)=\bar{N}(r, 1 /((1+\alpha) g-1))+S(r)$ and $\bar{N}(r, g)=\bar{N}(r, 1 /(\alpha f-$ $(\alpha+1)))+S(r)$. In the same manner as in Case 1, we get $(2.8)$.

Subcase 2.2. $\alpha \neq \beta$ and $\alpha=-1$. Consequently, from (2.7), we have $f=\beta /(-g+1+\beta)$ and $g=((\beta+1) f-\beta) / f$. In the same way as in Case 1 , we get (2.8). This proves that Lemmas 2.1 and 2.2 are clear when $H \equiv 0$.

Lemma 2.3 [5]. Let $f$ be a transcendental meromorphic function, and let $t, k$ be two positive integers. Then

$$
\begin{aligned}
& N_{t}\left(r, \frac{1}{f^{(k)}}\right) \leq T\left(r, f^{(k)}\right)-T(r, f)+N_{t+k}\left(r, \frac{1}{f}\right)+S(r, f), \\
& N_{t}\left(r, \frac{1}{f^{(k)}}\right) \leq k \bar{N}(r, f)+N_{t+k}\left(r, \frac{1}{f}\right)+S(r, f) .
\end{aligned}
$$

\section{Proofs of Theorems 1.6, 1.8, 1.9 and 1.11}

3.1. Proof of Theorems 1.6 and 1.8. Set $F=\alpha_{2} f^{n}\left(f^{m}+\alpha_{1}\right)$ and $G=\alpha_{2} g^{n}\left(g^{m}+\alpha_{1}\right)$. Suppose that $F^{(k)} \not \equiv G^{(k)}$ and $F^{(k)} G^{(k)} \not \equiv 1$, and condition (A1) in Theorem 1.6 holds. Then, by applying Lemma 2.1,

$$
\begin{aligned}
T\left(r, G^{(k)}\right) \leq N_{2}( & \left.r, F^{(k)}\right)+N_{2}\left(r, \frac{1}{F^{(k)}}\right)+N_{2}\left(r, G^{(k)}\right) \\
& +N_{2}\left(r, \frac{1}{G^{(k)}}\right)+2 \bar{N}\left(r, G^{(k)}\right)+2 \bar{N}\left(r, \frac{1}{G^{(k)}}\right) \\
& +\bar{N}\left(r, F^{(k)}\right)+\bar{N}\left(r, \frac{1}{F^{(k)}}\right)+S(r) .
\end{aligned}
$$


From this, and by applying Lemma 2.3 with $t=2$,

$$
\begin{aligned}
T(r, G) \leq & T\left(r, G^{(k)}\right)-N_{2}\left(r, \frac{1}{G^{(k)}}\right)+N_{k+2}\left(r, \frac{1}{G}\right)+S(r) \\
\leq & 3 \bar{N}(r, f)+4 \bar{N}(r, g)+N_{2}\left(r, \frac{1}{F^{(k)}}\right) \\
& \quad+2 \bar{N}\left(r, \frac{1}{G^{(k)}}\right)+\bar{N}\left(r, \frac{1}{F^{(k)}}\right)+N_{k+2}\left(r, \frac{1}{G}\right)+S(r) \\
\leq & (2 k+3) \bar{N}(r, f)+2(k+2) \bar{N}(r, g)+N_{k+1}\left(r, \frac{1}{F}\right)+N_{k+2}\left(r, \frac{1}{F}\right) \\
& \quad+2 N_{k+1}\left(r, \frac{1}{G}\right)+N_{k+2}\left(r, \frac{1}{G}\right)+S(r) \\
\leq & (2 k+3) \bar{N}(r, f)+2(k+2) \bar{N}(r, g)+2 m T(r, f)+3 m T(r, g)+N_{k+1}\left(r, \frac{1}{f^{n}}\right) \\
& \quad+N_{k+2}\left(r, \frac{1}{f^{n}}\right)+2 N_{k+1}\left(r, \frac{1}{g^{n}}\right)+N_{k+2}\left(r, \frac{1}{g^{n}}\right)+S(r) .
\end{aligned}
$$

By the assumption $n \geq k+1$, it is easy to show that $N_{k+2}\left(r, 1 / f^{n}\right) \leq(k+2) \bar{N}(r, 1 / f)+$ $S(r)$ and $N_{k+1}\left(r, 1 / f^{n}\right) \leq(k+1) \bar{N}(r, 1 / f)+S(r)$. Since $T(r, G)=(n+m) T(r, g)+$ $S(r)$, we deduce from (3.1) that

$$
\begin{array}{r}
(n-2 m) T(r, g) \leq(2 k+3) \bar{N}(r, f)+2(k+2) \bar{N}(r, g)+2 m T(r, f) \\
+(2 k+3) \bar{N}\left(r, \frac{1}{f}\right)+(3 k+4) \bar{N}\left(r, \frac{1}{g}\right)+S(r) .
\end{array}
$$

We observe that

$$
\bar{N}(r, f) \leq \frac{1}{t_{1}} N(r, f)+S(r) \leq \frac{1}{t_{1}} T(r, f)+S(r), \bar{N}\left(r, \frac{1}{f}\right) \leq \frac{1}{t_{2}} T(r, f)+S(r)
$$

and

$$
\bar{N}(r, g) \leq \frac{1}{t_{1}} T(r, g)+S(r), \bar{N}\left(r, \frac{1}{g}\right) \leq \frac{1}{t_{2}} T(r, g)+S(r) .
$$

It follows from these inequalities and (3.2) that

$$
\left(n-2 m-\frac{2 k+4}{t_{1}}-\frac{3 k+4}{t_{2}}\right) T(r, g) \leq\left(\frac{2 k+3}{t_{1}}+\frac{2 k+3}{t_{2}}+2 m\right) T(r, f)+S(r) .
$$

In the same way, we deduce that

$$
\left(n-2 m-\frac{2 k+4}{t_{1}}-\frac{3 k+4}{t_{2}}\right) T(r, f) \leq\left(\frac{2 k+3}{t_{1}}+\frac{2 k+3}{t_{2}}+2 m\right) T(r, g)+S(r) \text {. }
$$

The last two inequalities give us $(n-4 m) t_{1} t_{2} \leq(5 k+7) t_{1}+(4 k+7) t_{2}$, which contradicts condition (A1). By the same method, if $F^{(k)}$ and $G^{(k)}$ share $\left(1, \ell_{1}\right)^{*} \mathrm{CM}$ and $\left(1, \ell_{2}\right)^{*} \mathrm{IM}$, and condition (B1) or (B2) holds, then, by using Lemma 2.2, we will 
arrive at a contradiction. Therefore, we conclude that either $F^{(k)} \equiv G^{(k)}$ or $F^{(k)} G^{(k)} \equiv 1$. Suppose that

$$
F^{(k)} G^{(k)} \equiv 1
$$

We see that $m(r, 1 / F) \leq m(r, G)+S(r)$, which gives us

$$
\begin{aligned}
(n+m) T(r, f)+S(r) & =T(r, F) \leq T(r, G)+N\left(r, \frac{1}{F}\right)-N(r, G)+S(r) \\
& \leq T(r, G)+n N\left(r, \frac{1}{f}\right)+m T(r, f)-(n+m) N(r, g)+S(r) .
\end{aligned}
$$

We define the following sets:

$V_{1}=\left\{z: f(z)=0\right.$ of order $i(f, z)$ such that $\left.\alpha_{1}(z) \neq \infty, \alpha_{2}(z) \neq \infty\right\}$;

$V_{2}=\left\{z: f(z)=0\right.$ of order $i(f, z), \alpha_{1}(z) \neq \infty, \alpha_{2}(z)=\infty$ of order $i\left(\alpha_{2}, z\right)$ such that $\left.i\left(\alpha_{2}, z\right)<i(f, z)\right\}$;

$V_{3}=\left\{z: f(z)=0\right.$ of order $i(f, z), \alpha_{2}(z) \neq \infty, \alpha_{1}(z)=\infty$ of order $i\left(\alpha_{1}, z\right)$ such that $\left.i\left(\alpha_{1}, z\right)<i(f, z)\right\}$

$V_{4}=\left\{z: f(z)=0, \alpha_{1}(z)=\alpha_{2}(z)=\infty\right.$ of order $i(f, z), i\left(\alpha_{1}, z\right), i\left(\alpha_{2}, z\right)$ respectively, such that $\left.i\left(\alpha_{1}, z\right)+i\left(\alpha_{2}, z\right)<i(f, z)\right\}$.

Let $N_{V_{i}}(r)(i=1,2,3,4)$ denote the corresponding counting functions of the sets $V_{i}(i=1,2,3,4)$, respectively, and each point in these counting functions is counted according to its multiplicity of $f$. Obviously,

$$
N\left(r, \frac{1}{f}\right)=N_{V_{1}}(r)+N_{V_{2}}(r)+N_{V_{3}}(r)+N_{V_{4}}(r)+S(r) .
$$

We need to estimate $N_{V_{i}}(r), i=1,2,3,4$.

(1) Let $z \in V_{1}$, and let $i(f, z)=i(f)$ for short. We have the following cases.

(1-1) $\alpha_{2}(z) \neq 0$ and $\alpha_{1}(z)=0$ with multiplicity $i\left(\alpha_{1}\right)<i(f)$. Then $z$ must be a zero of $F^{(k)}$ with multiplicity $i\left(F^{(k)}\right)=n i(f)+i\left(\alpha_{1}\right)-k>1$. From (3.3), it follows that $z$ must be a pole of $g$ with multiplicity $i(g)$, and then $z$ will be a pole of $G^{(k)}$ with multiplicity $i\left(G^{(k)}\right)=(n+m) i(g)+k$. Equation (3.3) tells us that $i\left(G^{(k)}\right)=i\left(F^{(k)}\right)$, which is $n i(f)=(n+m) i(g)+2 k-i\left(\alpha_{1}\right)$.

(1-2) $\alpha_{1}(z) \neq 0$ and $\alpha_{2}(z)=0$ with multiplicity $i\left(\alpha_{2}\right)<i(f)$. Then $z$ must be a zero of $F^{(k)}$ with multiplicity $i\left(F^{(k)}\right)=n i(f)+i\left(\alpha_{2}\right)-k>1$. From (3.3), it follows that $z$ must be a pole of $g$ with multiplicity $i(g)$, and then $z$ will be a pole of $G^{(k)}$ with multiplicity $i\left(G^{(k)}\right)=(n+m) i(g)+k-i\left(\alpha_{2}\right)$. Equation (3.3) tells us that $i\left(G^{(k)}\right)=i\left(F^{(k)}\right)$, which is $n i(f)=(n+m) i(g)+2 k-2 i\left(\alpha_{2}\right)$.

(1-3) $\alpha_{1}(z)=0$ and $\alpha_{2}(z)=0$ with multiplicity $i\left(\alpha_{1}\right), i\left(\alpha_{2}\right)$, respectively, such that $i\left(\alpha_{1}\right)+i\left(\alpha_{2}\right)<i(f)$. Then $z$ must be a zero of $F^{(k)}$ with multiplicity $i\left(F^{(k)}\right)=n i(f)+$ $i\left(\alpha_{1}\right)+i\left(\alpha_{2}\right)-k>1$. From (3.3), it follows that $z$ must be a pole of $g$ with multiplicity $i(g)$, and then $z$ will be a pole of $G^{(k)}$ with multiplicity $i\left(G^{(k)}\right)=(n+m) i(g)+k-$ $i\left(\alpha_{2}\right)$. Equation (3.3) tells us that $i\left(G^{(k)}\right)=i\left(F^{(k)}\right)$, which is $n i(f)=(n+m) i(g)+2 k-$ $2 i\left(\alpha_{2}\right)-i\left(\alpha_{1}\right)$. 
(1-4) $\alpha_{1}(z) \neq 0$ and $\alpha_{2}(z) \neq 0$. Then, from (3.3), we deduce that $z$ must be a pole of $g$ with multiplicity $i(g)$ and $n i(f)=(n+m) i(g)+2 k$.

Consequently, from cases (1-1)-(1-4),

$$
n N_{V_{1}}(r) \leq(n+m) N_{V_{1}, g}(r)+2 k \overline{N_{V_{1}, g}}(r)+S(r),
$$

where $N_{V_{1}, g}(r)$ is the counting function of the poles of $g$ which belong to $V_{1}$, and $\overline{N_{V_{1}, g}}(r)$ is the reduced counting function of $N_{V_{1}, g}(r)$.

(2) Let $z \in V_{2}$, and let $i(f, z)=i(f)$ and $i\left(\alpha_{2}, z\right)=i\left(\alpha_{2}\right)$ for short. We have two cases.

(2-1) Suppose that $\alpha_{1}(z)=0$ with multiplicity $i\left(\alpha_{1}\right)<i(f)$. We observe that

$$
n i(f)-i\left(\alpha_{2}\right)=(n-1) i(f)+i(f)-i\left(\alpha_{2}\right) \geq(n-1) i(f)+1 \geq n \geq k+1,
$$

which means that $z$ is a zero of $F$ with multiplicity $n i(f)-i\left(\alpha_{2}\right)+i\left(\alpha_{1}\right)>k+1$, and then $z$ is a zero of $F^{(k)}$ with multiplicity $i\left(F^{(k)}\right)=n i(f)-i\left(\alpha_{2}\right)+i\left(\alpha_{1}\right)-k$. Then (3.3) tells us that $z$ is a pole of $G$.

If $g(z) \neq \infty$, then $z$ is a pole of $G^{(k)}$ with multiplicity less than or equal to $i\left(\alpha_{2}\right)+k$; from this and (3.3) it follows that $n i(f) \leq 2 i\left(\alpha_{2}\right)-i\left(\alpha_{1}\right)+2 k$. If $g(z)=\infty$ with multiplicity $i(g)$, then $z$ is a pole of $G^{(k)}$ with multiplicity $i\left(G^{(k)}\right)=(n+m) i(g)+k+$ $i\left(\alpha_{2}\right)$; from this and (3.3) it follows that $n i(f)=(n+m) i(g)+2 k+2 i\left(\alpha_{2}\right)-i\left(\alpha_{1}\right)$.

(2-2) Suppose that $\alpha_{1}(z) \neq 0$. In the same way as in case (2-1), we get that $z$ is a zero of $F^{(k)}$ with multiplicity $i\left(F^{(k)}\right)=n i(f)-i\left(\alpha_{2}\right)-k \geq 1$. Also, if $g(z) \neq \infty$, then $n i(f) \leq 2 i\left(\alpha_{2}\right)+2 k$, and if $g(z)=\infty$ with multiplicity $i(g)$, then $n i(f)=(n+m) i(g)+$ $2 k+2 i\left(\alpha_{2}\right)$.

Consequently, from cases (2-1) and (2-2),

$$
n N_{V_{2}}(r) \leq(n+m) N_{V_{2}, g}(r)+2 k \overline{N_{V_{2}, g}}(r)+S(r),
$$

where $N_{V_{2}, g}(r)$ is the counting function of the poles of $g$ which belong to $V_{2}$, and $\overline{N_{V_{2}, g}}(r)$ is the reduced counting function of $N_{V_{2}, g}(r)$.

(3) Let $z \in V_{3}$, and let $i(f, z)=i(f)$ and $i\left(\alpha_{1}, z\right)=i\left(\alpha_{1}\right)$ for short. We have two cases.

(3-1) Suppose that $\alpha_{2}(z)=0$ with multiplicity $i\left(\alpha_{2}\right)<i(f)$. In the same way as in case (2-1), we get that $z$ is a zero of $F^{(k)}$ with multiplicity $i\left(F^{(k)}\right)=n i(f)+i\left(\alpha_{2}\right)-$ $i\left(\alpha_{1}\right)-k \geq 1$. Also, if $g(z) \neq \infty$, then $n i(f) \leq 2 i\left(\alpha_{1}\right)-2 i\left(\alpha_{2}\right)+2 k$. Suppose that $g(z)=\infty$ with multiplicity $i(g)$.

If $i\left(\alpha_{1}\right)<m i(g)$, then $z$ is a pole of $G^{(k)}$ with multiplicity $i\left(G^{(k)}\right)=(n+m) i(g)+k-$ $i\left(\alpha_{2}\right)$; from this and (3.3) it follows that $n i(f)=(n+m) i(g)+2 k-2 i\left(\alpha_{2}\right)+i\left(\alpha_{1}\right)$.

If $m i(g) \leq i\left(\alpha_{1}\right)$, then $z$ is a pole of $G^{(k)}$ with multiplicity $i\left(G^{(k)}\right)$ and

$$
i\left(G^{(k)}\right) \leq n i(g)+k-i\left(\alpha_{2}\right)+i\left(\alpha_{1}\right) \leq\left(\frac{n}{m}+1\right) i\left(\alpha_{1}\right)-i\left(\alpha_{2}\right)+k .
$$

Thus, $n i(f) \leq(n / m+2) i\left(\alpha_{1}\right)-2 i\left(\alpha_{2}\right)+2 k$.

(3-2) Suppose that $\alpha_{2}(z) \neq 0$. In the same way as in case (2-1), we get that $z$ is a zero of $F^{(k)}$ with multiplicity $i\left(F^{(k)}\right)=n i(f)-i\left(\alpha_{1}\right)-k \geq 1$. If $g(z) \neq \infty$, then $n i(f) \leq 2 i\left(\alpha_{1}\right)+2 k$. Suppose that $g(z)=\infty$ with multiplicity $i(g)$. 
If $i\left(\alpha_{1}\right)<m i(g)$, then $z$ is a pole of $G^{(k)}$ with multiplicity $i\left(G^{(k)}\right)=(n+m) i(g)+k$; from this and (3.3) it follows that $n i(f)=(n+m) i(g)+2 k+i\left(\alpha_{1}\right)$.

If $m i(g) \leq i\left(\alpha_{1}\right)$, we proceed as in case (3-1) to get $n i(f) \leq(n / m+2) i\left(\alpha_{1}\right)+2 k$.

Consequently, from (3-1) and (3-2),

$$
n N_{V_{3}}(r) \leq(n+m) N_{V_{3}, g}(r)+2 k \overline{N_{V_{3}, g}}(r)+S(r),
$$

where $N_{V_{3}, g}(r)$ is the counting function of the poles of $g$ which belong to $V_{3}$, and $\overline{N_{V_{3}, g}}(r)$ is the reduced counting function of $N_{V_{3}, g}(r)$.

(4) Let $z \in V_{4}$, and let $i(f, z)=i(f), i\left(\alpha_{1}, z\right)=i\left(\alpha_{1}\right)$ and $i\left(\alpha_{2}, z\right)=i\left(\alpha_{2}\right)$ for short. We see that

$$
n i(f)-i\left(\alpha_{1}\right)-i\left(\alpha_{2}\right)=(n-1) i(f)+i(f)-i\left(\alpha_{1}\right)-i\left(\alpha_{2}\right) \geq(n-1) i(f)+1 \geq k+1,
$$

that is, $z$ is a zero of $F$ with multiplicity $n i(f)-i\left(\alpha_{2}\right)-i\left(\alpha_{1}\right) \geq k+1$, and then $z$ is a zero of $F^{(k)}$ with multiplicity $i\left(F^{(k)}\right)=n i(f)-i\left(\alpha_{2}\right)-i\left(\alpha_{1}\right)-k$. If $g(z) \neq \infty$, then $n i(f) \leq 2 i\left(\alpha_{1}\right)+2 i\left(\alpha_{2}\right)+2 k$.

Suppose that $g(z)=\infty$ with multiplicity $i(g)$.

If $i\left(\alpha_{1}\right)<m i(g)$, then $z$ is a pole of $G^{(k)}$ with multiplicity $i\left(G^{(k)}\right)=(n+m) i(g)+k+$ $i\left(\alpha_{2}\right)$; from this and (3.3) it follows that $n i(f)=(n+m) i(g)+2 k+i\left(\alpha_{1}\right)+2 i\left(\alpha_{2}\right)$.

If $m i(g) \leq i\left(\alpha_{1}\right)$, then we proceed as in case (3-1) to get

$$
n i(f) \leq(n / m+2) i\left(\alpha_{1}\right)+2 i\left(\alpha_{2}\right)+2 k .
$$

Consequently,

$$
n N_{V_{4}}(r) \leq(n+m) N_{V_{4}, g}(r)+2 k \overline{N_{V_{4}, g}}(r)+S(r),
$$

where $N_{V_{4}, g}(r)$ is the counting function of the poles of $g$ which belong to $V_{4}$, and $\overline{N_{V_{4}, g}}(r)$ is the reduced counting function of $N_{V_{4}, g}(r)$.

By using the estimates (3.5)-(3.9),

$$
n N\left(r, \frac{1}{f}\right) \leq(n+m) N(r, g)-(n+m) N_{f \neq 0}(r, g)+2 k \bar{N}\left(r, \frac{1}{f}\right)+S(r),
$$

where $N_{f \neq 0}(r, g)$ is the counting function of those poles of $g$ which are not zeros of $f$.

Let $z$ be a zero of $f$ of order $q$ such that $z$ is not any zero or pole of $\alpha_{1}$ or $\alpha_{2}$. We deduce from (3.3) that $z$ must be a pole of $g$ of order $p$ with $n q=(n+m) p+2 k$. We see that $n(q-p)=m p+2 k \geq n$, and we deduce that $q \geq p+1 \geq 1+((n-2 k) / m)$. Therefore, $\bar{N}(r, 1 / f) \leq m /(m+n-2 k) N(r, 1 / f)+S(r)$; from this, (3.4) and (3.10), it follows that

$$
n T(r, f)+(n+m) s_{g} \bar{N}_{f \neq 0}(r, g) \leq(n+m) T(r, g)+2 k \bar{N}\left(r, \frac{1}{f}\right)+S(r),
$$

where $\bar{N}_{f \neq 0}(r, g)$ is the reduced counting function of $N_{f \neq 0}(r, g)$. 
From the last inequality,

$$
\begin{aligned}
n T(r, f)+(n+m) s_{g} \bar{N}(r, g) & \leq(n+m) T(r, g)+\left(\left((n+m) s_{g}\right)+2 k\right) \bar{N}\left(r, \frac{1}{f}\right)+S(r) \\
& \leq(n+m) T(r, g)+m \frac{2 k+(n+m) s_{g}}{n+m-2 k} N\left(r, \frac{1}{f}\right)+S(r) \\
& \leq(n+m) T(r, g)+m \frac{2 k+(n+m) s_{g}}{n+m-2 k} T(r, f)+S(r),
\end{aligned}
$$

which implies that

$$
\left(\frac{n-2 k-m s_{g}}{n+m-2 k}\right) T(r, f) \leq T(r, g)-s_{g} \bar{N}(r, g)+S(r) .
$$

Similarly,

$$
\left(\frac{n-2 k-m s_{f}}{n+m-2 k}\right) T(r, g) \leq T(r, f)-s_{f} \bar{N}(r, f)+S(r) .
$$

The last two inequalities lead to

$$
\limsup _{r \rightarrow \infty} \frac{s_{g} \bar{N}(r, g)+s_{f} \bar{N}(r, f)}{\left(1+s_{g}\right) T(r, f)+\left(1+s_{f}\right) T(r, g)} \leq \frac{m}{n+m-2 k},
$$

in contradiction to condition (A2), and this contradiction comes from the hypothesis $F^{(k)} G^{(k)} \equiv 1$. Suppose that $F^{(k)} \equiv G^{(k)}$.

Consequently, $F+\delta=G$ and $T(r, f)=T(r, g)+S(r)$, where $\delta$ is a polynomial. On the other hand, if $\delta \not \equiv 0$, by applying Nevanlinna's three small functions theorem (see $[8$, Theorem 1.36]), we see that

$$
\begin{aligned}
(n+m) T(r, f) & \leq \bar{N}(r, f)+\bar{N}\left(r, \frac{1}{\alpha_{2} f^{n}\left(f^{m}+\alpha_{1}\right)}\right)+\bar{N}\left(r, \frac{1}{\alpha_{2} g^{n}\left(g^{m}+\alpha_{1}\right)}\right)+S(r) \\
& \leq\left(\frac{1}{t_{1}}+\frac{2}{t_{2}}+2 m\right) T(r, f)+S(r),
\end{aligned}
$$

giving us $(n-m) t_{1} t_{2} \leq 2 t_{1}+t_{2}$, which contradicts condition (A1) (condition (B1) or condition (B2)). Hence, $\delta \equiv 0$, and $F \equiv G$.

Let $n=q n_{1}, m=q m_{1}, f / g=h$ and $h^{q}=H$, where $q=\operatorname{gcd}(m, n)$. Then

$$
g^{m}=-\alpha_{1} \frac{H^{n_{1}}-1}{H^{n_{1}+m_{1}}-1} \quad \text { and } \quad f^{n}\left(f^{m}+\alpha_{1}\right) \equiv g^{n}\left(g^{m}+\alpha_{1}\right) .
$$

Suppose that $H$ is a nonconstant meromorphic function. Since $n>m$ (from conditions (A1), (B1) and (B2)), we have $n_{1} \geq 2$. However, from (3.11) and the Valiron-Mokhon'ko lemma,

$$
m T(r, g)=\left(n_{1}+m_{1}-1\right) T(r, H)+S(r) .
$$


Let $\lambda_{1}, \ldots, \lambda_{n_{1}+m_{1}-1}$ be the distinct complex numbers satisfying $\lambda_{i} \neq 1$ and $\lambda_{i}^{n_{1}+m_{1}}=$ $1\left(1 \leq i \leq n_{1}+m_{1}-1\right)$, and let $\xi_{1}, \ldots, \xi_{n_{1}-1}$ be the distinct complex numbers satisfying $\xi_{i} \neq 1$ and $\xi_{i}^{n_{1}}=1\left(1 \leq i \leq n_{1}-1\right)$. We note that $\lambda_{1}, \ldots, \lambda_{n_{1}+m_{1}-1}, \xi_{1}, \ldots, \xi_{n_{1}-1}$ are distinct; it follows from (3.11) and (3.12), and by applying the second fundamental theorem, that

$$
\begin{aligned}
\left(2 n_{1}+m_{1}-3\right) T(r, H) \leq & \sum_{i=1}^{n_{1}+m_{1}-1} \bar{N}\left(r, \frac{1}{H-\lambda_{i}}\right)+\sum_{i=1}^{n_{1}-1} \bar{N}\left(r, \frac{1}{H-\xi_{i}}\right) \\
& +\bar{N}(r, H)+S(r, H) \\
\leq & \bar{N}(r, g)+\bar{N}\left(r, \frac{1}{g}\right)+S(r) \leq \frac{1}{t_{1}} N(r, g)+\frac{1}{t_{2}} N\left(r, \frac{1}{g}\right) \\
& +S(r) \leq \frac{2}{t} T(r, g)+S(r),
\end{aligned}
$$

where $t=2 t_{1} t_{2} /\left(t_{1}+t_{2}\right)$.

From (3.12) and (3.13) we deduce $m t\left(2 n_{1}+m_{1}-3\right) \leq 2\left(n_{1}+m_{1}-1\right)$, which can be written as

$$
2(n+m-q)(m t-1) \leq m t(m+q) .
$$

We claim that $n>2 q$. This argument is clear when condition (A1) holds. Suppose that $n \leq 2 q$ under condition (B1) or (B2). If $n=2$, then $m=q=1$, and then (3.14) becomes $t_{1} t_{2} \leq t_{1}+t_{2}$, but from condition (B1) or (B2) we have $t_{1} t_{2}>6 t_{1}+5 t_{2}$, which is impossible. Thus, $n>2$, and we get $n_{1}=2$ and $m=q$ (because $n>m$ ). This means that $n=2 q$, and inequality (3.14) implies $q t_{1} t_{2} \leq t_{1}+t_{2}$, and from this and condition (B1) (or condition (B2)) we see that

$$
(k+4) t_{2}+2(k+2) t_{1}<(n-m) t_{1} t_{2}<n t_{1} t_{2} \leq 2\left(t_{1}+t_{2}\right),
$$

which is impossible. This shows that $n>2 q$.

We note that $m+q<n+m-q$; if $m t \leq 2(m t-1)$ then $m t(m+q)<2(n+m-$ $q)(m t-1)$, which contradicts (3.14). Therefore, we have $m t<2$, which implies $m t_{1} t_{2}<t_{1}+t_{2}$; from this inequality we deduce that $m=1$ and $t_{1} t_{2}<t_{1}+t_{2}$, and from the last inequality we get that either $t_{1}=1$ or $t_{2}=1$. Suppose that $t_{1}=1$. Therefore, inequality (3.14) becomes $n(t-1) \leq t$, which means that $n\left(t_{2}-1\right) \leq 2 t_{2}$. We observe that if condition (A1) holds then $n \geq 5$, which is impossible unless $t_{2}=1$. Suppose that $t_{2} \geq 2$ and condition (B1) or (B2) holds. Therefore, from condition (B1) or (B2) we have $6+5 t_{2}<(n-1) t_{2}$; if we compare this inequality with $n\left(t_{2}-1\right) \leq 2 t_{2}$, we will arrive at a contradiction. Therefore, we have $m=t_{1}=t_{2}=1$, and condition (A3) holds. It follows from (3.12) and (3.13) that

$$
\frac{2 n-2}{n} T(r, g) \leq \bar{N}(r, g)+\bar{N}\left(r, \frac{1}{g}\right)+S(r) .
$$


By the same method,

$$
\frac{2 n-2}{n} T(r, f) \leq \bar{N}(r, f)+\bar{N}\left(r, \frac{1}{f}\right)+S(r) .
$$

Then inequalities (3.15) and (3.16) will give a contradiction with condition (A3). Therefore, we conclude that $H$ must be a constant; it follows from (3.11) that $H^{n_{1}} \equiv H^{n_{1}+m_{1}} \equiv 1$, which means that $H \equiv 1$, which means in turn that $h$ is a constant, and hence $f=c g$, where $c$ is a nonzero constant satisfying $c^{q}=1$. This finishes the proofs of Theorems 1.6 and 1.8 .

3.2. Proof of Theorem 1.9. Set $F=\alpha_{1} f^{n}$ and $G=\alpha_{2} g^{n}$. Suppose that $F^{(k)} \not \equiv G^{(k)}$ and $F^{(k)} G^{(k)} \not \equiv 1$. We proceed as in the proof of inequality (3.2) to get

$$
\begin{aligned}
& n T(r, g) \leq(2 k+3) \bar{N}(r, f)+2(k+2) \bar{N}(r, g) \\
& \quad+(2 k+3) \bar{N}\left(r, \frac{1}{f}\right)+(3 k+4) \bar{N}\left(r, \frac{1}{g}\right)+S(r),
\end{aligned}
$$

which implies that

$$
\left(n-2 \frac{k+2}{t_{1}}-\frac{3 k+4}{t_{2}}\right) T(r, g) \leq\left(\frac{2 k+3}{t_{1}}+\frac{2 k+3}{t_{2}}\right) T(r, f)+S(r) .
$$

In the same way, we deduce that

$$
\left(n-2 \frac{k+2}{t_{1}}-\frac{3 k+4}{t_{2}}\right) T(r, f) \leq\left(\frac{2 k+3}{t_{1}}+\frac{2 k+3}{t_{2}}\right) T(r, g)+S(r) .
$$

These two inequalities give us $n t_{1} t_{2} \leq(5 k+7) t_{1}+(4 k+7) t_{2}$, which contradicts condition (C1). Therefore, from Lemma 2.1, we conclude that either $F^{(k)} \equiv G^{(k)}$ or $F^{(k)} G^{(k)} \equiv 1$.

Suppose that $F^{(k)} \equiv G^{(k)}$. Consequently, $F+\delta=G$ and $T(r, f)=T(r, g)+S(r)$, where $\delta$ is a polynomial. On the other hand, if $\delta \neq 0$, by applying Nevanlinna's three small functions theorem we get

$$
n T(r, f) \leq \bar{N}(r, f)+\bar{N}\left(r, \frac{1}{\alpha_{1} f^{n}}\right)+\bar{N}\left(r, \frac{1}{\alpha_{2} g^{n}}\right)+S(r) \leq\left(\frac{1}{t_{1}}+\frac{2}{t_{2}}\right) T(r, f)+S(r),
$$

leading to $n t_{1} t_{2} \leq 2 t_{1}+t_{2}$, which contradicts condition (C1). Hence, $\delta \equiv 0$, and then $\alpha_{1} f^{n} \equiv \alpha_{2} g^{n}$, which is also a contradiction. Therefore,

$$
F^{(k)} G^{(k)} \equiv 1
$$

If $z$ is a zero of $f$ of multiplicity $q$ such that $z$ is not any pole or zero of any element in $\phi$ or $\alpha$ then, from (3.17), that $z$ must be a pole of $g$ of order $p$ with $n q-k=n p+k$, which means that $n(q-p)=2 k \geq n$, which is impossible. This proves that $\bar{N}(r, 1 / f)=S(r)$. Similarly, $\bar{N}(r, 1 / g)=S(r)$. 
Let $z_{0}$ be a pole of $f$ with multiplicity $p \geq t_{1}$ such that $z_{0}$ is not any zero of $G$ or any function in $\alpha$ or $\phi$. Consequently, from (3.17), we deduce that $z_{0}$ is a zero of $G^{(k)}$ with multiplicity $n p+k \geq n t_{1}+k$. Therefore, by the lemma of the logarithmic derivative, we observe that

$$
\begin{aligned}
\bar{N}(r, f) & \leq \frac{1}{n t_{1}+k} N\left(r, \frac{G^{(k)}}{G}\right)+S(r) \\
& \leq \frac{1}{n t_{1}+k}\left(k \bar{N}(r, G)+N_{k}\left(r, \frac{1}{G}\right)\right)+S(r) \leq \frac{k}{n t_{1}+k} \bar{N}(r, g)+S(r) .
\end{aligned}
$$

Similarly, $\bar{N}(r, g) \leq\left(k /\left(n t_{1}+k\right)\right) \bar{N}(r, f)+S(r)$. Consequently, we conclude from the last two inequalities that $\bar{N}(r, f) \leq\left(k /\left(n t_{1}+k\right)\right)^{2} \bar{N}(r, f)+S(r)$, which leads to $\bar{N}(r, f)=S(r)$ and $\bar{N}(r, g)=S(r)$.

We take the $k$ th derivative of $F$ and $G$ to get $F^{(k)}=\lambda_{1} f^{n}$ and $G^{(k)}=\lambda_{2} g^{n}$, where $\lambda_{1}$ and $\lambda_{2}$ are small functions of $f$ and $g$. It follows from this and (3.17) that $f^{n}=a g^{-n}$, where $a=\left(\lambda_{1} \lambda_{2}\right)^{-1}$.

We now turn to the last part of Theorem 1.9 when $\alpha$ and $\phi$ consist of only complex constants. Then $\alpha_{1}$ and $\alpha_{2}$ are constants. As noted in the paragraph after (3.17), if $z$ is a zero of $f$ of multiplicity $q$, then $z$ must be a pole of $g$ of order $p$ with $n q-k=n p+k$, which means that $n(q-p)=2 k \geq n$, which is impossible. Therefore, $f$ has no zeros, and in the same way we get that $g$ has no zeros. This proves Theorem 1.9.

In the same manner as in the above proof and by using Lemma 2.2 we prove Theorem 1.11.

\section{References}

[1] R. S. Dyavanal, 'Uniqueness and value-sharing of differential polynomials of meromorphic functions', J. Math. Anal. Appl. 374 (2011), 335-345.

[2] M. L. Fang, 'Uniqueness and value-sharing of entire functions', Comput. Math. Appl. 44 (2002), $828-831$.

[3] W. K. Hayman, Meromorphic Functions (Clarendon Press, Oxford, 1964).

[4] S. H. Lin and W. C. Lin, 'Uniqueness of meromorphic functions concerning weakly weightedsharing', Kodai Math. J. 29 (2006), 269-280.

[5] W. C. Lin and H. X. Yi, 'Uniqueness theorems for meromorphic functions', Indian J. Pure Appl. Math. 35 (2004), 121-132.

[6] C. Wu, C. Mu and J. Li, 'Uniqueness of meromorphic functions sharing one value', Bull. Aust. Math. Soc. 85 (2012), 280-294.

[7] C. C. Yang and X. H. Hua, 'Uniqueness and value-sharing of meromorphic functions', Ann. Acad. Sci. Fenn. Math. 22 (1997), 395-406.

[8] C. C. Yang and H. X. Yi, Uniqueness Theory of Meromorphic Functions (Kluwer Academic Publishers, Dordrecht, 2003).

THAMIR ALZAHARY, Korteweg-de Vries Institute for Mathematics, Amsterdam University, PO BOX 94248, Amsterdam 1090GE, The Netherlands e-mail: thammra@yahoo.com 